CTP TAMU-17/95

$\mathrm{UG}-2 / 95$

hep-th/9504140

April 1995

\title{
Super $p$-Brane Theories and New Spacetime Superalgebras
}

\author{
E. Bergshoeff ${ }^{1}$ and E. Sezgin ${ }^{2} \dagger$ \\ ${ }^{1}$ Institute for Theoretical Physics, Nijenborgh 4, 9747 AG Groningen, The Netherlands. \\ ${ }^{2}$ Center for Theoretical Physics, Texas A\&M University, College Station, TX 77843-4242, \\ USA.
}

\begin{abstract}
We present a geometric formulation of super $p$-brane theories in which the Wess-Zumino term is $(p+1)$-th order in the supersymmetric currents, and hence is manifestly supersymmetric. The currents are constructed using a supergroup manifold corresponding to a generalization of a superalgebra which we found sometime ago. Our results generalize Siegel's analogous reformulation of the Green-Schwarz superstring. The new superalgebras we construct underly the free differential superalgebras introduced by de Azcárraga and Townsend a few years ago.
\end{abstract}

\footnotetext{
$\dagger$ Supported in part by the National Science Foundation, under grant PHY-9411543.
} 


\section{Introduction}

The dynamics of super $p$-brane theories is notoriously difficult. Therefore, it is useful to look for simplifications and/or alternative formulations of these theories. Often a phenomenon that arises in the Green-Schwarz superstring generalizes to super $p$-branes as well, which suggests that there is a universal structure in a handful theories known as super $p$ branes in $d$ dimensions, which exist for $d \leq 11$ and $p \leq 5$. Indeed, here we find yet another parallel between superstrings and higher super $p$-branes.

Less than a year ago, Siegel [1] found a manifestly supersymmetric formulation of the Green-Schwarz superstring, based on a superalgebra discovered earlier by Green [2]. The algebra generalizes the super Poincaré algebra in that it contains a new fermionic generator, and that translations do not commute with the supercharges. Siegel constructed a suitable set of currents on the corresponding group manifold, and succeeded in writing the WessZumino term of the Green-Schwarz action in a manifestly supersymmetric form, without having to go to one higher dimension. He also showed why this new formulation was useful in the lattice formulation of the theory.

In this paper, we show that Siegel's formulation generalizes to higher super $p$-branes. To do this, we introduce a set of new spacetime superalgebras, including central charge generators, whose extension is based on the same gamma-matrix identities that underly the super $p$-brane theories. In other words, to every super $p$-brane corresponds a new spacetime superalgebra. This set of algebras is intimately related to the super $p$-brane loop algebras we found before [3]. Using the new algebras, we first construct manifestly supersymmetric currents in the supergroup space. We next use these currents to write down a Wess-Zumino term for super $p$-branes as $(p+1)$-th order expressions in the supercurrents, without the need to extend the $(p+1)$-dimensional worldvolume to a space of one dimension higher. Thus, by introducing the new coordinates corresponding to the new generators of the underlying superalgebra, we are able to write the Wess-Zumino term in a form which is manifestly supersymmetric and which equals the usual Wess-Zumino term up to total derivative terms.

The organization of this letter is as follows. For pedagogical reasons we first review Siegel's new formulation of the superstring. In section 3 we shall focus our attention on the case of supermembranes since this case already covers all the essential features necessary to proceed to the higher super $p$-brane case. In particular, we will present the new spacetime superalgebra corresponding to the supermembrane and construct the supercurrents and supersymmetry transformations. We will then present the new formulation of the supermembrane action. Next, in section 4 we will discuss the generalization to higher super $p$-branes. Finally, in the conclusions we will compare the new formulation presented here with the description, first introduced by de Azcárraga and Townsend, of super $p$-brane theories based upon free differential superalgebras [4]. 


\section{Superstrings}

The starting point in Siegel's new formulation [1] of the superstring is the following superalgebra introduced by Green [2]:

$$
\begin{aligned}
\left\{Q_{\alpha}, Q_{\beta}\right\} & =\Gamma_{\alpha \beta}^{\mu} P_{\mu}, \\
{\left[P_{\mu}, Q_{\alpha}\right] } & =-\left(\Gamma_{\mu}\right)_{\alpha \beta} \Sigma^{\beta},
\end{aligned}
$$

where $\Sigma^{\beta}$ is a new fermionic generator. The Jacobi-identities corresponding to the algebra (2.1) require the gamma-matrix identity

$$
\Gamma_{(\alpha \beta}^{\mu} \Gamma_{\gamma) \delta}^{\mu}=0
$$

which is only valid in $d=3,4,6,10$ dimensional spacetimes. For definiteness we consider the ten dimensional superstring. The spinor-index $\alpha$ labels a 16 component Majorana-Weyl spinor $^{\dagger}$, and $\mu=0,1, \ldots, 10$.

The supergroup manifold corresponding to the superalgebra (2.1) is parametrized as follows:

$$
U=e^{\phi_{\alpha} \Sigma^{\alpha}} e^{x^{\mu} P_{\mu}} e^{\theta^{\alpha} Q_{\alpha}}
$$

where we have introduced the coordinates $Z^{M}=\left(\phi_{\alpha}, x^{\mu}, \theta^{\alpha}\right)$ which correspond to the generators $T_{A}=\left(\Sigma^{\alpha}, P_{\mu}, Q_{\alpha}\right)$, respectively. In order to calculate the supercurrents we consider the following decomposition of the left-invariant Maurer-Cartan form $U^{-1} d U$ :

$$
U^{-1} \partial_{i} U=\partial_{i} Z^{M} L_{M}{ }^{A} T_{A}=L_{i}{ }^{A} T_{A}
$$

A straightforward calculation leads to the following expressions for the pull-backs $L_{i}{ }^{A}$ of the left-invariant group vielbeins $L_{M}{ }^{A}$ :

$$
\begin{aligned}
L_{i}^{\alpha} & =\partial_{i} \theta^{\alpha}, \quad L_{i}^{\mu}=\partial_{i} x^{\mu}+\frac{1}{2} \bar{\theta} \Gamma^{\mu} \partial_{i} \theta \\
L_{i \alpha} & =\partial_{i} \phi_{\alpha}-\partial_{i} x^{\mu}\left(\Gamma_{\mu} \theta\right)_{\alpha}-\frac{1}{6}\left(\Gamma_{\mu} \theta\right)_{\alpha} \bar{\theta} \Gamma^{\mu} \partial_{i} \theta .
\end{aligned}
$$

Similarly, we can define the right-invariant group vielbeins $R_{M}{ }^{A}$ as follows

$$
\partial_{i} U U^{-1}=\partial_{i} Z^{M} R_{M}^{A} T_{A}=R_{i}^{A} T_{A}
$$

The left-group transformations, which leave the pull-backs $L_{i}{ }^{A}$ invariant then take the form

$$
\delta Z^{M}=\epsilon^{A} R_{A}^{M}
$$

$\dagger$ We use a chiral notation where the position of the spinor-index indicates the chirality. In case we do not denote the spinor indices explicitly, it is always understood that they have their standard position, e.g. $\left(\Gamma_{\mu} \theta\right)_{\alpha}=\left(\Gamma_{\mu}\right)_{\alpha \beta} \theta^{\beta}, \bar{\theta} \Gamma^{\mu} \partial_{i} \theta=\theta^{\alpha}\left(\Gamma^{\mu}\right)_{\alpha \beta} \partial_{i} \theta^{\beta}$, etc. 
where $\epsilon^{A}$ are constant parameters. In particular, the transformation parameter $\epsilon^{\alpha}$ can be interpreted as the rigid supersymmetry transformation parameter. One finds that these supersymmetry transformations are given by*

$$
\begin{gathered}
\delta \theta^{\alpha}=\epsilon^{\alpha}, \quad \delta x^{\mu}=-\frac{1}{2} \bar{\epsilon} \Gamma^{\mu} \theta, \\
\delta \phi_{\alpha}=x^{\mu}\left(\Gamma_{\mu} \epsilon\right)_{\alpha}-\frac{1}{6}\left(\bar{\epsilon} \Gamma^{\mu} \theta\right)\left(\Gamma_{\mu} \theta\right)_{\alpha} .
\end{gathered}
$$

Note that the supercovariant derivatives can be viewed as

$$
D_{A}=L_{A}^{M} \partial_{M}
$$

while the supersymmetry generators $Q_{A}$ which commute with these derivatives can be written as

$$
Q_{A}=R_{A}^{M} \partial_{M}
$$

In Siegel's formulation, the superstring action is written as follows

$$
I \text { (string })=\int d^{2} \zeta\left[-\frac{1}{2} \sqrt{-\gamma} \gamma^{i j} L_{i}^{\mu} L_{j \mu}-\frac{1}{2} \epsilon^{i j} L_{i}^{\alpha} L_{j \alpha}\right]
$$

where $\gamma_{i j}$ is the worldsheet metric and $\gamma=\operatorname{det} \gamma_{i j}$. The nontrivial feature of the new action is that the new coordinate $\phi_{\alpha}$ only occurs as a total derivative. Up to this total derivative term the above action is identical to the standard GreenSchwarz action. Furthermore, the supersymmetry of the Wess-Zumino term in the above action is manifest, unlike in the usual Green-Schwarz formulation where the supersymmetry is up to a total derivative term.

The action (2.11) is also invariant under the usual $\kappa$-symmetry transformations. These transformations involve $L_{i}^{\mu}$ and $L_{i}^{\alpha}$ which remain unchanged by the presence of the new coordinate $\phi_{\alpha}$, as can be seen from (2.5).

The Wess-Zumino term in the action (2.11) suggests the definition of a two-form $B$ according to

$$
B=\frac{1}{2} L_{\alpha} \wedge L^{\alpha}
$$

where the left-invariant basis one-forms $L^{A}$ are defined by $L^{A}=d Z^{M} L_{M}{ }^{A}$. Then, using the Maurer-Cartan equation

$$
d L^{A}-\frac{1}{2} L^{B} \wedge L^{C} f_{C B}{ }^{A}=0,
$$

one finds that the field strength $H=d B$ is given by

* Note the presence of the bare $x^{\mu}$ in the formula below. It turns out that the theory is still translation invariant though not so manifestly. 


$$
H=\frac{1}{2} L^{\beta} \wedge L^{\alpha} \wedge L^{a} \Gamma_{a \alpha \beta},
$$

which is the same expression one finds in the usual formulation of the superstring. Note that all dependence on the new coordinates have dropped out from the expression of $H$.

In the next section we will generalize the above construction to the case of supermembrane theories while in section 4 we will discuss the general $p$-brane case. For the generic $p$-brane the Poincaré superalgebra needs to be extended with both fermionic as well as as bosonic generators. In hindsight, it turns out that the superstring case is special in the sense that the general $p$-brane case predicts, for $p=1$, a new bosonic generator, $\Sigma^{\mu}$, that can be redefined away into the translation generator $P_{\mu}$ via $P_{\mu}^{\prime}=P_{\mu}+\Sigma^{\mu}$.

\section{The New Supermembrane Action}

Supermembranes exist in $d=4,5,7,11$ dimensional spacetimes [5,6]. Therefore, we need to consider the generalizations of the the corresponding super Poincaré algebras. Here, for definiteness let us consider the eleven dimensional supermembrane [5]. The supersymmetry and translation generators are $Q_{\alpha}$ and $P_{\mu}$, respectively, where $\alpha$ labels a 32 component Majorana spinor ${ }^{\dagger}$, and $\mu=0,1, \ldots 10$. Sometime ago we introduced the additional generators $\Sigma^{\mu \nu}$ and $\Sigma^{\mu \alpha}$ and wrote down a generalization of the super Poincaré algebra [3]. In trying to generalize Siegel's new formulation of the Green-Schwarz string to higher $p$-branes, it turns out that we need to further extend the algebra of [3] by introducing also the bosonic generators $\Sigma^{\alpha \beta}=\Sigma^{\beta \alpha}$. The new superalgebra we have found takes the following form ${ }^{\ddagger}$ :

$$
\begin{aligned}
\left\{Q_{\alpha}, Q_{\beta}\right\} & =\Gamma_{\alpha \beta}^{\mu} P_{\mu}+\left(\Gamma_{\mu \nu}\right)_{\alpha \beta} \Sigma^{\mu \nu} \\
{\left[P_{\mu}, Q_{\alpha}\right] } & =-\left(\Gamma_{\mu \nu}\right)_{\alpha \beta} \Sigma^{\nu \beta} \\
{\left[P_{\mu}, P_{\nu}\right] } & =\left(\Gamma_{\mu \nu}\right)_{\alpha \beta} \Sigma^{\alpha \beta} \\
{\left[P_{\mu}, \Sigma^{\lambda \tau}\right] } & =\frac{1}{2} \delta_{\mu}^{[\lambda} \Gamma_{\alpha \beta}^{\tau]} \Sigma^{\alpha \beta} \\
{\left[Q_{\alpha}, \Sigma^{\mu \nu}\right] } & =\left(\Gamma^{[\mu}\right)_{\alpha \beta} \Sigma^{\nu] \beta} \\
\left\{Q_{\alpha}, \Sigma^{\nu \beta}\right\} & =\left(\frac{1}{4} \Gamma_{\gamma \delta}^{\nu} \delta_{\alpha}^{\beta}+2 \Gamma_{\gamma \alpha}^{\nu} \delta_{\delta}^{\beta}\right) \Sigma^{\gamma \delta} .
\end{aligned}
$$

To verify the Jacobi-identities one needs to use the well-known gamma-matrix identity:

$$
\Gamma_{(\alpha \beta}^{\mu \nu} \Gamma_{\gamma \delta)}^{\nu}=0
$$

\footnotetext{
$\dagger \quad$ In the calculations we never need to raise or lower a spinor index using the charge-conjugation matrix. It is convenient to use a notation where a given spinor always has an upper or a lower spinor-index, e.g. $Q_{\alpha}, \Sigma^{\mu \beta}, \theta^{\alpha}$, etc. In case we do not denote the spinor indices explicitly, it is always understood that they have their standard position, e.g. $\left(\Gamma_{\mu} \theta\right)_{\alpha}=\left(\Gamma_{\mu}\right)_{\alpha \beta} \theta^{\beta}, \bar{\theta} \Gamma^{\mu} \partial_{i} \theta=\theta^{\alpha}\left(\Gamma^{\mu}\right)_{\alpha \beta} \partial_{i} \theta^{\beta}$, etc.

$\ddagger \quad$ The first line of (3.1) also occurs in [7] where it was derived by looking to the total derivative terms in the supersymmetry variation of the Wess-Zumino term in the standard Green-Schwarz action.
} 
The algebra of [3] corresponds to a Wigner-Inönü contraction of the above algebra in which one rescales $\Sigma^{\alpha \beta} \rightarrow c \Sigma^{\alpha \beta}$ and then takes the limit $c \rightarrow 0$.

We now turn to the construction of various geometrical objects on the corresponding supergroup manifold. Since the calculations are more involved than in the superstring case it is convenient to first present some general formulae. Denoting a group element generically by $U=e^{\phi}$, the exterior derivative of $\phi$ by $d \phi$ and a super Lie algebra valued entity by $\beta$, the following formulae [8] are useful in the computations that will follow:

$$
e^{\phi} \beta e^{-\phi}=e^{\phi} \wedge \beta, \quad e^{\phi} d e^{-\phi}=\left(\frac{1-e^{\phi}}{\phi}\right) \wedge d \phi
$$

where we have used the notation

$$
\phi \wedge \beta \equiv[\phi, \beta], \quad \phi^{2} \wedge \beta \equiv \phi \wedge \phi \wedge \beta=[\phi,[\phi, \beta]], \quad \text { etc. }
$$

Note that the wedge $(\wedge)$ notation used here denotes an operation involving multiple commutators, and is not to be confused with the exterior product for forms. It turns out that in the following we need at most the triple-commutator terms in (3.3).

Next, we need to parametrize our supergroup manifold. A suitable parametrization takes the form ${ }^{\dagger}$

$$
U=e^{\phi_{\mu \nu} \Sigma^{\mu \nu}} e^{\phi_{\mu \alpha} \Sigma^{\mu \alpha}} e^{\phi_{\alpha \beta} \Sigma^{\alpha \beta}} e^{x^{\mu} P_{\mu}} e^{\theta^{\alpha} Q_{\alpha}}
$$

where we have introduced the coordinates

$$
Z^{M}=\left(\phi_{\mu \nu}, \phi_{\mu \alpha}, \phi_{\alpha \beta}, x^{\mu}, \theta^{\alpha}\right)
$$

which correspond to the generators

$$
T_{A}=\left(\Sigma^{\mu \nu}, \Sigma^{\mu \alpha}, \Sigma^{\alpha \beta}, P_{\mu}, Q_{\alpha}\right)
$$

respectively.

We first calculate the left-invariant Maurer-Cartan form $U^{-1} d U$, which we have been referring to as supercurrents above. Armed with the parametrization (3.5) and the formulae (3.3), it is straightforward to calculate the left-currents $L_{i}{ }^{A}$ defined in (2.4), for which we

$\dagger \quad$ Note that this parametrization is not of the form $U=e^{\phi}$. Due to this, the second equation in (3.3) is not enough to calculate the supercurrents, we also need the first equation. 
find the following results:

$$
\begin{aligned}
L_{i}^{\alpha} & =\partial_{i} \theta^{\alpha}, \quad L_{i}^{\mu}=\partial_{i} x^{\mu}+\frac{1}{2} \bar{\theta} \Gamma^{\mu} \partial_{i} \theta \\
L_{i \mu \nu} & =\partial_{i} \phi_{\mu \nu}+\frac{1}{2} \bar{\theta} \Gamma_{\mu \nu} \partial_{i} \theta \\
L_{i \mu \alpha} & =\partial_{i} \phi_{\mu \alpha}+\partial_{i} \phi_{\mu \nu}\left(\Gamma^{\nu} \theta\right)_{\alpha}+\partial_{i} x^{\nu}\left(\Gamma_{\mu \nu} \theta\right)_{\alpha}+\frac{1}{6}\left(\Gamma_{\mu \nu} \theta\right)_{\alpha} \bar{\theta} \Gamma^{\nu} \partial_{i} \theta+\frac{1}{6}\left(\Gamma^{\nu} \theta\right)_{\alpha} \bar{\theta} \Gamma_{\mu \nu} \partial_{i} \theta, \\
L_{i \alpha \beta} & =\partial_{i} \phi_{\alpha \beta}-\frac{1}{2} x^{\mu} \partial_{i} \phi_{\mu \nu}\left(\Gamma^{\nu}\right)_{\alpha \beta}+\partial_{i} \phi_{\mu \nu}\left(\Gamma^{\mu} \theta\right)_{(\alpha}\left(\Gamma^{\nu} \theta\right)_{\beta)}+\frac{1}{4}\left(\bar{\theta} \partial_{i} \phi_{\mu}\right)\left(\Gamma^{\mu}\right)_{\alpha \beta} \\
& +2\left(\Gamma^{\mu} \theta\right)_{(\alpha} \partial_{i} \phi_{\mu \beta}-\frac{1}{2} x^{\mu} \partial_{i} x^{\nu}\left(\Gamma_{\mu \nu}\right)_{\alpha \beta}-\left(\Gamma^{\nu} \theta\right)_{(\alpha}\left(\Gamma_{\mu \nu} \theta\right)_{\beta)} \partial_{i} x^{\mu} \\
& -\frac{1}{12}\left(\Gamma_{\nu} \theta\right)_{(\alpha}\left(\Gamma^{\mu \nu} \theta\right)_{\beta)}\left(\bar{\theta} \Gamma_{\mu} \partial_{i} \theta\right)-\frac{1}{12}\left(\Gamma_{\nu} \theta\right)_{(\alpha}\left(\Gamma_{\mu} \theta\right)_{\beta)}\left(\bar{\theta} \Gamma^{\mu \nu} \partial_{i} \theta\right) .
\end{aligned}
$$

Note that $L_{i}^{\alpha}$ and $L_{i}^{\mu}$ take the same form as they do in the usual superspace.

The supersymmetry transformations are defined in (2.7). A convenient way to calculate them is as follows. We first write the finite transformation as $\left(e^{\phi}\right)^{\prime}=e^{\epsilon} e^{\phi}$, where $\epsilon=\epsilon^{A} T_{A}$ is the parameter and $e^{\phi}=e^{Z \cdot T}$ is the group element. We next observe that the infinitesimal transformation can be written as $(1+\epsilon) e^{\phi}=e^{\phi+\delta \phi}$. Multiplying from the left by $e^{-\phi}$ we obtain $e^{-\phi} \epsilon e^{\phi}=e^{-\phi} e^{\phi+\delta \phi}-1=e^{-\phi} \delta e^{\phi}$. Using (3.3) we then find the formula

$$
e^{-\phi} \wedge \epsilon=\left(\frac{1-e^{-\phi}}{\phi}\right) \wedge \delta \phi
$$

from which one can solve for $\delta Z^{M}$. Again we only need at most the triple-commutator terms which are given by $^{\dagger}$ :

$$
\delta Z \cdot T=\epsilon \cdot T-\frac{1}{2}[Z \cdot T, \epsilon \cdot T]+\frac{1}{12}[Z \cdot T,[Z \cdot T, \epsilon \cdot T]]+\cdots .
$$

After some algebra, using (3.3), we obtain the following result for the the supersymmetry

$\dagger$ Note that the coefficient of the triple-commutator term vanishes identically. 
transformations (with parameter $\epsilon^{\alpha}$ ):

$$
\begin{aligned}
\delta \theta^{\alpha}= & \epsilon^{\alpha}, \quad \delta x^{\mu}=-\frac{1}{2} \bar{\epsilon} \Gamma^{\mu} \theta \\
\delta \phi_{\mu \nu}= & -\frac{1}{2} \bar{\epsilon} \Gamma_{\mu \nu} \theta, \\
\delta \phi_{\mu \alpha}= & -x^{\nu}\left(\Gamma_{\mu \nu} \epsilon\right)_{\alpha}-\phi_{\mu \nu}\left(\Gamma^{\nu} \epsilon\right)_{\alpha}, \\
& +\frac{1}{6}\left(\bar{\epsilon} \Gamma_{\mu \nu} \theta\right)\left(\Gamma^{\nu} \theta\right)_{\alpha}+\frac{1}{6}\left(\bar{\epsilon} \Gamma^{\nu} \theta\right)\left(\Gamma_{\mu \nu} \theta\right)_{\alpha}, \\
\delta \phi_{\alpha \beta}= & -\frac{1}{4}\left(\Gamma^{\mu}\right)_{\alpha \beta} \bar{\epsilon} \phi_{\mu}-2\left(\Gamma^{\mu} \epsilon\right)_{(\alpha} \phi_{\mu \beta} \\
& -\frac{1}{4} x^{\mu}\left(\bar{\epsilon} \Gamma_{\mu \nu} \theta\right)\left(\Gamma^{\nu}\right)_{\alpha \beta}-\frac{1}{4} x^{\mu}\left(\bar{\epsilon} \Gamma^{\nu} \theta\right)\left(\Gamma_{\mu \nu}\right)_{\alpha \beta} \\
& \left.-\frac{1}{12} \bar{\epsilon} \Gamma_{\mu \nu} \theta\left(\Gamma^{\mu} \theta\right)_{(\alpha}\left(\Gamma^{\nu} \theta\right)_{\beta)}-\frac{1}{12} \bar{\epsilon} \Gamma_{\mu} \theta\left(\Gamma^{\mu \nu} \theta\right)_{(\alpha}\left(\Gamma_{\nu} \theta\right)_{\beta}\right) .
\end{aligned}
$$

We are now in a position to write down a manifestly supersymmetric action for the supermembrane. Using the expressions (3.8) we find the following new action:

$$
\begin{aligned}
I(\text { membrane })=\int d^{3} \zeta & {\left[-\frac{1}{2} \sqrt{-\gamma} \gamma^{i j} L_{i}^{\mu} L_{j \mu}+\frac{1}{2} \sqrt{-\gamma}\right.} \\
& \left.-\epsilon^{i j k}\left(L_{i}^{\mu} L_{j}^{\nu} L_{k \mu \nu}+\frac{9}{10} L_{i}^{\mu} L_{j}^{\alpha} L_{k \mu \alpha}-\frac{1}{5} L_{i}^{\alpha} L_{j}^{\beta} L_{k \alpha \beta}\right)\right],
\end{aligned}
$$

where $\gamma_{i j}$ is the worldvolume metric and $\gamma=\operatorname{det} \gamma_{i j}$. The last three terms in the action constitute the manifestly supersymmetric form of the Wess-Zumino term. The coefficients in front of these terms are nontrivially fixed in such a way that all possible structures with the new coordinates in them, i.e. with $\phi_{\mu \nu}, \phi_{\mu \alpha}$ and $\phi_{\alpha \beta}$ are total derivative terms. Like in the superstring case, the action (3.12) is invariant under the usual kappa-symmetry transformations, the new coordinates being inert.

The Wess-Zumino term in the membrane action (3.12) leads us to define a three-form $B$ as follows:

$$
B=L^{\mu} \wedge L^{\nu} \wedge L_{\mu \nu}+\frac{9}{10} L^{\mu} \wedge L^{\alpha} \wedge L_{\mu \alpha}-\frac{1}{5} L^{\alpha} \wedge L^{\beta} \wedge L_{\alpha \beta}
$$

Using the structure equations (2.13), where the structure constants are given by (3.1), we find that the field strength $H=d B$ is given by

$$
H=\frac{3}{2} L^{\mu} \wedge L^{\nu} \wedge L^{\alpha} \wedge L^{\beta} \Gamma_{\mu \nu \alpha \beta}
$$

Note that, just as in the string case, the field strength $H$ takes exactly the same form that it does in the usual Green-Schwarz formulation. This is not surprising since, as mentioned 
earlier, the very construction of the new supermembrane action is such that all the dependence on the new coordinates is contained in total derivative terms. In fact, the problem of finding the new formulation of a super $p$-brane reduces to that of finding a $(p+1)-$ form whose field strengh, calculated by making use of the Maurer-Cartan equations of the new spacetime superalgebras, takes the same form as the one that arises in the ordinary formulation of the super $p$-branes [5].

Finally, we note that in order to find a three-form $B$ trilinear in the line-elements $L$ such that its field-strength $H=d B$ is given by by (3.14), i.e. does not depend on the new coordinates, it is essential that we introduce the extra generator $\Sigma^{\alpha \beta}$. Without it, the Ansatz for $B$ would only involve the first two terms of (3.13) which now refer to the membrane algebra (3.1) with $\Sigma^{\alpha \beta}=0$. One can easily verify that for that case it is not possible to define a $B$ whose field-strength is given by (3.14). In conclusion, in order to write down the new membrane action one needs to introduce all generators $\Sigma^{\mu \nu}, \Sigma^{\mu \alpha}$ and $\Sigma^{\alpha \beta}$.

\section{Generalization to Higher Super $p-$ Branes}

Our basic interest in this section is to construct an extension of the Poincare superalgebra and a corresponding $p+1$-form $B$ which is $(p+1)$ th-order in the line-elements of the algebra and whose field-strength $H=d B$ is identical to the expression of the usual Green-Schwarz formulation. It is not difficult to generalize the membrane algebra (3.1) to general $p$. It is convenient to characterize the $p$-brane analog of the membrane algebra (3.1) by giving the Maurer-Cartan structure equations. For general $p$ they are given by:

$$
\begin{aligned}
d L^{\mu} & =\frac{1}{2} L^{\alpha} \wedge L^{\beta} \Gamma_{\alpha \beta}^{\mu}, \quad d L^{\alpha}=0, \\
d L_{\mu_{1} \cdots \mu_{p}} & =\frac{1}{2} L^{\alpha} \wedge L^{\beta}\left(\Gamma_{\mu_{1} \cdots \mu_{p}}\right)_{\alpha \beta}, \\
d L_{\mu_{1} \cdots \mu_{p-1} \alpha} & =-L^{\beta} \wedge L^{\nu}\left(\Gamma_{\nu \mu_{1} \cdots \mu_{p-1}}\right)_{\beta \alpha}-L^{\beta} \wedge L_{\nu \mu_{1} \cdots \mu_{p-1}} \Gamma_{\beta \alpha}^{\nu}, \\
d L_{\mu_{1} \cdots \mu_{p-2} \alpha \beta} & =\frac{1}{2} L^{\nu} \wedge L^{\rho}\left(\Gamma_{\rho \nu \mu_{1} \cdots \mu_{p-2}}\right)_{\alpha \beta}+\frac{1}{2} L_{\mu \nu \mu_{1} \cdots \mu_{p-2}} \wedge L^{\mu} \Gamma_{\alpha \beta}^{\nu} \\
& +\frac{1}{4} L_{\nu \mu_{1} \cdots \mu_{p-2} \gamma} \wedge L^{\gamma} \Gamma_{\alpha \beta}^{\nu}+2 L_{\nu \mu_{1} \cdots \mu_{p-2}(\alpha} \wedge L^{\gamma} \Gamma_{\beta) \gamma}^{\nu} .
\end{aligned}
$$

The verification of the Jacobi-identities or, equivalently, the integrability conditions $d^{2} L=0$ of the above structure equations require the usual $p$-brane gamma-matrix identities

$$
\left(\Gamma_{\mu_{1} \cdots \mu_{p}}\right)_{(\alpha \beta} \Gamma_{\gamma \delta)}^{\mu_{p}}=0 .
$$

In order to explain how the extension to the general $p$-brane case goes, we first consider the generalization from $p=2$ to $p=3$. Our aim is to construct a four-form $B$ whose 
field-strength $H=d B$ does only depend on $L^{\mu}$ and $L^{\alpha}$. Given the algebra corresponding to (4.1) (taken for $p=3$ ) the most general Ansatz for $B$ would be ${ }^{\dagger}$ :

$$
B=L^{\mu} \wedge L^{\nu} \wedge L^{\rho} \wedge L_{\mu \nu \rho}+\alpha_{1} L^{\mu} \wedge L^{\nu} \wedge L^{\alpha} \wedge L_{\mu \nu \alpha}+\alpha_{2} L^{\mu} \wedge L^{\alpha} \wedge L^{\beta} \wedge L_{\mu \alpha \beta}
$$

with $\alpha_{1}, \alpha_{2}$ arbitrary coefficients. However, one would soon discover that for no choice of $\alpha_{1}, \alpha_{2}$ it is possible to construct the desired $B$. In fact, it turns out that one needs to extend the algebra further with a generator $\Sigma^{\alpha \beta \gamma}$ which has the special property that it occurs as a central charge. This is analogous to the status of the generator $\Sigma^{\alpha \beta}$ in the membrane algebra $(3.1)^{\ddagger}$. It turns out that the appropriately extended algebra is characterized by the structure equations (4.1), taken with $p=3$, together with the following new structure equation:

$$
d L_{\alpha \beta \gamma}=L^{\nu} \wedge L_{\mu \nu(\alpha} \Gamma_{\beta \gamma)}^{\mu}-\frac{5}{2} L^{\delta} \wedge L_{\mu(\alpha \beta} \Gamma_{\gamma) \delta}^{\mu}-\frac{1}{2} L^{\delta} \wedge L_{\mu \delta(\alpha} \Gamma_{\beta \gamma)}^{\mu},
$$

where we have fixed the normalization of $\Sigma^{\alpha \beta \gamma}$.

For completeness, we give the commutation relations of the $p=3$ superalgebra below:

$$
\begin{aligned}
\left\{Q_{\alpha}, Q_{\beta}\right\} & =\Gamma_{\alpha \beta}^{\mu} P_{\mu}+\left(\Gamma_{\mu \nu \rho}\right)_{\alpha \beta} \Sigma^{\mu \nu \rho}, \\
{\left[P_{\mu}, Q_{\alpha}\right] } & =-\left(\Gamma_{\mu \nu \rho}\right)_{\alpha \beta} \Sigma^{\nu \rho \beta} \\
{\left[P_{\mu}, P_{\nu}\right] } & =\left(\Gamma_{\mu \nu \rho}\right)_{\alpha \beta} \Sigma^{\rho \alpha \beta}, \\
{\left[P_{\mu}, \Sigma^{\nu \rho \lambda}\right] } & =\frac{1}{2} \delta_{\mu}^{[\nu} \Gamma_{\alpha \beta}^{\rho} \Sigma^{\lambda] \alpha \beta}, \\
{\left[Q_{\alpha}, \Sigma^{\mu \nu \rho}\right] } & =\left(\Gamma^{[\mu}\right)_{\alpha \beta} \Sigma^{\nu \rho] \beta} \\
\left\{Q_{\alpha}, \Sigma^{\nu \rho \beta}\right\} & =\left(\frac{1}{4} \Gamma_{\gamma \delta}^{[\nu} \delta_{\alpha}^{\beta}+2 \Gamma_{\gamma \alpha}^{[\nu} \delta_{\delta}^{\beta}\right) \Sigma^{\rho] \gamma \delta}, \\
{\left[P_{\mu}, \Sigma^{\nu \rho \alpha}\right] } & =\delta_{\mu}^{[\nu} \Gamma_{\beta \gamma}^{\rho]} \Sigma^{\alpha \beta \gamma}, \\
{\left[Q_{\alpha}, \Sigma^{\mu \beta \gamma}\right] } & =\left(\frac{1}{2} \Gamma_{\delta \epsilon}^{\mu} \delta_{\alpha}^{(\beta}+\frac{5}{2} \Gamma_{\delta \alpha}^{\mu} \delta_{\epsilon}^{(\beta)}\right) \Sigma^{\gamma) \delta \epsilon} .
\end{aligned}
$$

We find that, with respect to the new spacetime superalgebra (4.5), it is possible to write down the desired $B$. The explicit expression is given by

$$
\begin{aligned}
B= & L^{\mu} \wedge L^{\nu} \wedge L^{\rho} \wedge L_{\mu \nu \rho}-\frac{87}{70} L^{\mu} \wedge L^{\nu} \wedge L^{\alpha} \wedge L_{\mu \nu \alpha} \\
& -\frac{36}{70} L^{\mu} \wedge L^{\alpha} \wedge L^{\beta} \wedge L_{\mu \alpha \beta}+\frac{6}{70} L^{\alpha} \wedge L^{\beta} \wedge L^{\gamma} \wedge L_{\alpha \beta \gamma} .
\end{aligned}
$$

The corresponding field-strength $H$ is given by

\footnotetext{
$\dagger \quad$ Note that $B$ is only determined up to a gauge transformation $\delta B=d \lambda$. The expression we find for $B$ is however unique if we also require that $B$ is written in terms of (products of) line-elements only.

$\ddagger$ Note that the generator $\Sigma^{\mu \alpha \beta}$ also occurs as a central charge generator in the algebra (4.1). However, as we will see below, this ceases to be true after the inclusion of the new generators $\Sigma^{\alpha \beta \gamma}$.
} 


$$
H=d B=-2 L^{\mu} \wedge L^{\nu} \wedge L^{\rho} \wedge L^{\alpha} \wedge L^{\beta}\left(\Gamma_{\mu \nu \rho}\right)_{\alpha \beta} .
$$

and indeed only depends on $L^{\mu}$ and $L^{\alpha}$, as it should be.

It is now clear how the generalization to the higher $p$-brane case goes. One first notices that the extended algebra characterized by (4.1) and (4.4) can be easily extended to general values of $p$ in the same way as the membrane algebra (3.1) was generalized to (4.1). However, for the next case $p=4$ this extended algebra will not be enough to construct a five-form $B$ with the desired properties. One needs to extend even further the algebra given by (4.1), (4.4) (taken for $p=4$ ) by including a new generator $\Sigma^{\alpha_{1} \cdots \alpha_{4}}$ which in the new algebra occurs as a central charge generator and satisfies a structure equation similar to (4.4). Having completed the $p=4$-case one then repeats the above procedure to get the final $p=5$-case. The precise expressions of the $p=4,5$-algebras are not very illuminating and will be given elsewhere $[9]$.

\section{Concluding Remarks}

The discussion in the previous section shows that the required extended algebra underlying the new formulation of the super $p$-brane contains the generators ${ }^{\star}$

$$
\begin{aligned}
P_{\mu}, Q_{\alpha} & \rightarrow P_{A}, \\
\Sigma^{\mu_{1} \cdots \mu_{p}}, \Sigma^{\mu_{1} \cdots \mu_{p-1} \alpha}, \cdots, \Sigma^{\alpha_{1} \cdots \alpha_{p}} & \rightarrow \Sigma^{A_{1} \cdots A_{p}} .
\end{aligned}
$$

Such an algebra corresponds to a supergroup manifold with coordinates

$$
\left\{Z^{M}, A_{M_{1} \cdots M_{p}}\right\}
$$

The same supergroup manifold was introduced in [4] and, more recently, considered in the context of a scale-invariant formulation of superstrings [10] and super $p$-branes [11].

More specifically, in the scale-invariant formulation one introduces a (world-volume) $p+1$-form

$$
F(A, Z)=d A+B^{\prime}(Z)
$$

where the $p$-form $B^{\prime}(Z)$ is a given expression in terms of $Z^{M}$ that corresponds to the WessZumino term in the standard super $p$-brane action. One can show that the supersymmetry variation of $B$ is given by $\delta_{\epsilon} B^{\prime}(Z)=d(\bar{\epsilon} \Delta(Z))$ for some $p$-form $\Delta(Z)$ which is a given expression in terms of $Z^{M}$. The $p$-form $F(A, Z)$ given in (5.3) will then be invariant if $\delta_{\epsilon} A=$ $\bar{\epsilon} \Delta(Z)$ and can be used to construct an action with manifest space-time supersymmetry.

The $p+1$-form $F(A, Z)$ satisfies the structure equation

\footnotetext{
* For simplictly of notation, from now on we shall let $A=\mu, \alpha$ and, similarly, $Z^{M}=\left(x^{\mu}, \theta^{\alpha}\right)$.
} 


$$
d F(A, Z)=c_{p} L^{\mu_{1}} \wedge \cdots \wedge L^{\mu_{p}} \wedge L^{\alpha} \wedge L^{\beta}\left(\Gamma_{\mu_{1} \cdots \mu_{p}}\right)_{\alpha \beta},
$$

where $c_{p}$ is a $p$-dependent constant. This structure equation, together with the structure equations defining the Poincaré superalgebra, i.e. the first line of (4.1), defines an extension of the Poincaré superalgebra which is called a free differential superalgebra or Cartan integrable system [12].

The concept of a free differential algebra was originally introduced to describe supergravity theories containing higher-rank antisymmetric tensors [13]. The idea is that the MaurerCartan equations (2.13) have a natural extension to the case where the line-elements $L$ do not only represent one-forms but general $p$-forms. The system is integrable if $d^{2} L=0$. Sometimes a free differential superalgebra is equivalent to an underlying Lie superalgebra but not always. Whether or not this is true for the free differential superalgebra discussed above depends on the following. With respect to the ordinary Poincaré superalgebra the $p+2$-form $H=d F$ is closed, i.e. $d H=0$ but not exact, i.e. $H$ cannot be written as $H=d B$ where $B$ is a $p+1$-form written as a product of the basic one-forms $\left(L^{a}, L^{\alpha}\right)^{\dagger}$. This means that $H$ belongs to a non-trivial class of the $(p+2)$-th Chevalley-Eilenberg cohomology group $[12,4]$. The question whether there is a Lie superalgebra underlying the free differential superalgebra is equivalent to the question whether or not the Poincaré superalgebra can be extended in such a way that the closed $p+2$-form $H$ is exact with respect to the extended algebra [12]. In this letter we have shown that this is indeed the case and we have given the explicit form of the spacetime superalgebras that underly the free differential superalgebras of de Azcárraga and Townsend [4].

It is interesting to compare the case of the eleven-dimensional supermembrane [5] with that of eleven-dimensional supergravity. In both cases there exists a description in terms of a free differential superalgebra. In the case of eleven-dimensional supergravity it has been shown [13] that the free differential superalgebra is equivalent to an extension of the Poincaré superalgebra containing additional bosonic generators $\Sigma^{\mu \nu}, \Sigma^{\mu_{1} \cdots \mu_{5}}$ and a fermionic generator $Q^{\prime}$. In this letter we have shown that the free differential algebra underlying the supermembrane is equivalent to an extension of the Poincaré superalgebra with the additional bosonic generators $\Sigma^{\mu \nu}, \Sigma^{\alpha \beta}$ and a fermionic generator $\Sigma^{\mu \alpha}$. It would be interesting to see in which sense the extension of the Poincaré superalgebra is unique and whether or not it is possible to obtain the same extended superalgebra both for eleven-dimensional supergravity and the eleven-dimensional supermembrane. This would be natural in view of the fact that eleven-dimensional supergravity is supposed to describe the low-energy limit of the eleven-dimensional supermembrane.

It is suggestive to rewrite the new spacetime superalgebras we have introduced using superspace notation. The $\Gamma^{\mu}$ matrices in the structure constants would then correspond to

$\dagger \quad$ Note that the $p$-form $B^{\prime}(Z)$ corresponding to the usual Wess-Zumino term always contains a bare $x^{\mu}$ and/or $\theta$ and therefore is not of the required form. 
torsion components $T_{B C}{ }^{A}$ while the $\Gamma_{\mu_{1} \cdots \mu_{p}}$-matrices would correspond to specific components of the $H$-tensor $H_{A_{1} \cdots A_{p+2}}$. However, so far we have not been able to write our results into this form. In fact, the results of [3] suggest that in order to do so one would need an underlying loop algebra instead of the ordinary Lie-superalgebras considered in this paper.

The new superalgebras presented in this letter may be relevant to the construction of the Yang-Mills coupled fivebrane action which is sometimes referred to as the heterotic fivebrane. It may also exhibit hidden duality symmetries of the kind that have been elusive so far in the usual sigma-model formulation of super $p$-brane theories. In particular, the new bosonic coordinate $y_{\mu_{1} \cdots \mu_{p}}$ may play a significant role.

It would be interesting to investigate the consequences of the new formulation of the supermembrane theory in its quantization program. Relevant to this question is the Hamiltonian formulation of the results of this paper and the generalization to curved superspace. These issues will be addressed elsewhere.

Finally, it is our hope that the new algebras presented in this letter will be useful in making progress in some of the outstanding open problems in super $p$-brane theories.

\section{ACKNOWLEDGMENTS}

E.S. would like to thank the Institute for Theoretical Physics in Groningen for hospitality. The work of E.B. has been made possible by a fellowship of the Royal Netherlands Academy of Arts and Sciences (KNAW). 


\section{REFERENCES}

1. W. Siegel, Phys. Rev. D50 (1994) 2799.

2. M.B. Green, Phys. Lett. B223 (1989) 157.

3. E. Bergshoeff and E. Sezgin, Phys. Lett. B232 (1989) 96.

4. J.A. de Azcárraga and P.K. Townsend, Phys. Rev. Lett. 62 (1989) 2579.

5. E. Bergshoeff, E. Sezgin and P.K. Townsend, Phys. Lett. 189B (1987) 75.

6. A. Achúcarro, J.M. Evans, P.K. Townsend and D.L. Wiltshire, Phys. Lett. 198B (1987) 441.

7. J.A. de Azcárraga, J.P. Gauntlett, J.M. Izquierdo and P.K. Townsend, Phys. Rev. Lett. 63 (1989) 2443.

8. B. Zumino, Nucl. Phys. B127 (1977) 189.

9. E. Bergshoeff and E. Sezgin, in preparation.

10. J.A. de Azcárraga, J.M. Izquierdo and P.K. Townsend, Phys. Rev. D45 (1992) 3321; P.K. Townsend, Phys. Lett. B277 (1992) 285.

11. E. Bergshoeff, L.A.J. London and P.K. Townsend, Class. Quantum Grav. 9 (1992) 2545.

12. For an introduction to Cartan integrable systems, see L. Castellani, P. Fré, F. Gianni, K. Pilch and P. van Nieuwenhuizen, Ann. of Phys. 146 (1983) 35.

13. R. D'Auria and P. Fré, Nucl. Phys. B201 (1982) 101. 